\title{
Transformation of Uml Class Diagram to UmI Sequence Diagram
}

\author{
Lavleen Kambow \\ Department of Computer Science \\ G.N.D.E.C., Ludhiana, India.
}

\begin{abstract}
For object-oriented software development, The Unified Modeling Language (UML) has become an industrial standard. There are different models which have been described under UML notation. Use case model can represent the functional view of the system, UML class model can represent static view of the system, activity or sequence diagram can represent the dynamic view of the system. If we focus on field of UML class diagram \& UML activity diagrams then a lots of research still is required. In this paper we describe a transformation process which can be used to transform the UML class diagram to UML sequence diagram. Transformation process is quite helpful so that we can easy obtain the UML sequence diagram from UML class diagram.
\end{abstract}

\section{General Terms}

UML Class diagram, UML Sequence diagram

\section{Keywords}

Transformation process, transformation algorithm.

\section{INTRODUCTION}

A class diagram is a type of static structure diagram which is used to represent the structure of a system. This static structure can be represented by presenting the system's classes, their attributes, methods and operations, the relationships among the classes [1], [11]. This UML class diagrams can be transformed/mapped directly with object oriented a language that is why; they are widely used in the modeling of object oriented systems [5], [9]. In contrast, the UML Sequence diagram is a kind of interaction diagram that shows in which order and how processes operate with one another .It is also known as a Message Sequence Chart. In this objects interact with each other in a timely fashion [1], [4], [7]. It represents the different various objects and classes involved in the system scenario and the sequence of messages exchanged between the objects needed to carry out the functionality of the scenario. Sequence diagrams are associated with use case realizations in the Logical View of the system under development [4], [12]. There are a many algorithms which can be used for transforming the UML models to the other form, like the transformation of UML model to Petri Net, here generally the state chart and collaboration, activity diagrams are used as an input for the transformation algorithm, which will give us Petri Net in the form of Constraints-based Modular Petri Nets, Colored Petri Nets, Extended Colored Petri Nets, and High-Level Timed Petri Nets [6], [10], [3]. But there is not such technique or process is invented which can help us to transform the UML class diagram to UML sequence diagram.

\section{TRANSFORMATION PROCESS}

There is much software available like Umbrello software IBM Rational Software Architect, which can be used to draw any UML model, but these applications are not used to transform the UML class diagram to UML sequence diagram. Let us discuss the step by step phenomenon for transforming the UML Class Diagram to UML Sequence Diagram [2], [4].

\subsection{Install the software requirement}

Install a software that can help us to draw an UML class diagram on your personal computer, so install IBM Rational Software Architect for this purpose [6], when the IBM Rational Software Architect get installed then make an UML Modeling Project, say "class diagram" as shown in figure 1.1

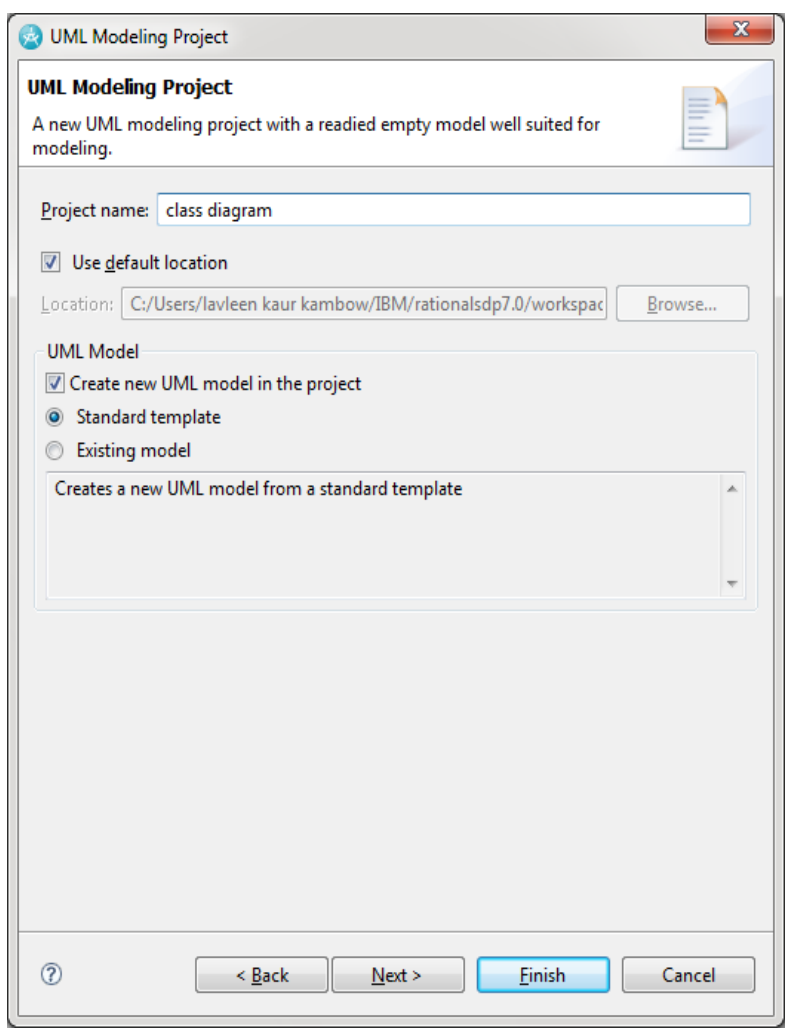

Fig 1: Uml Modeling Project 


\subsection{Create the UML Class Diagram}

Create the UML class diagram say of "Order management" in IBM Rational Software Architect for any system which we want to transform to UML Sequence Diagram, use available tools of IBM Rational Software Architect to draw the Class Diagram of "Order management system" [12].

\subsection{Export}

IBM Rational Software Architect is well known for its features like model-to-code and code to model transformations/mapping. Model-to-code transformation/mapping is called forward /transformation mapping and code-to-model transformation/ mapping is called the reverse transformation/ mapping [4], [7]. Export the UML class diagram to our personal computer in any suitable language [1], [5]. We will follow the UML to XML forward approach for export process. Select the export destination say "UML Model" as shown in figure 2.

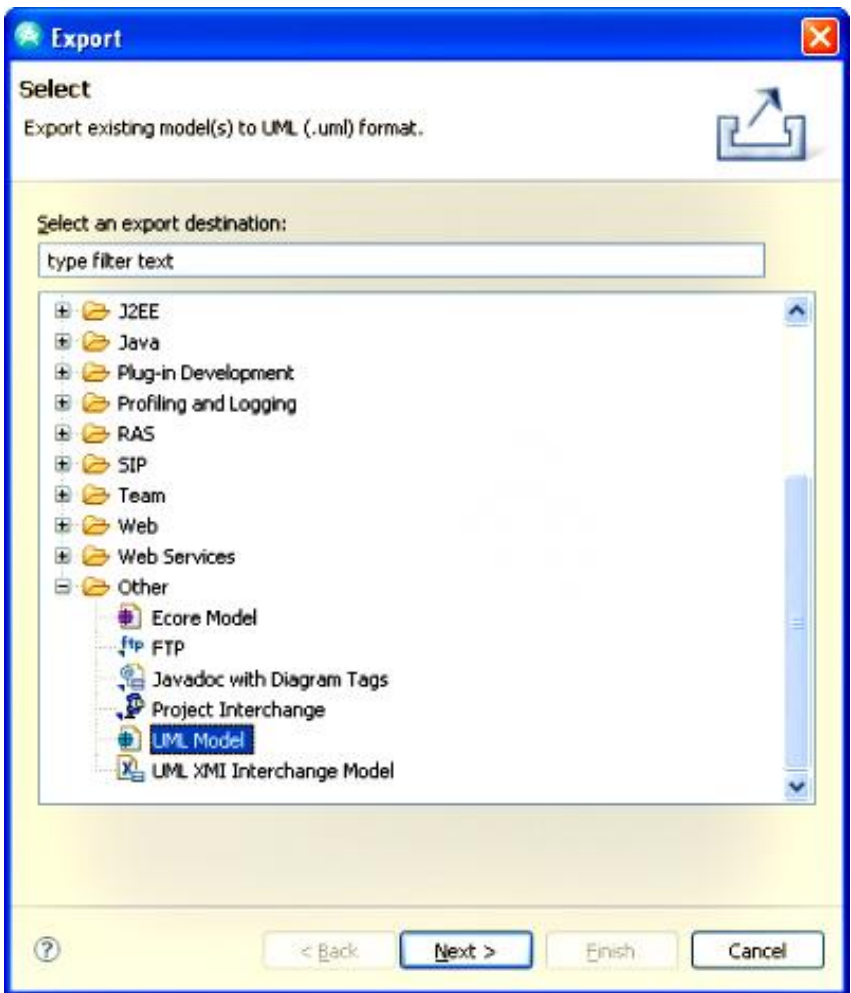

Fig 2: Exporting model to UML format

\subsection{XML metadata interface form}

After export process, we get a file consists the UML coding of the class diagram in the form of XML metadata interface which has its own structure. Now apply transformation algorithm this file to get the transformation from UML Class Diagram to UML Sequence Diagram [10], [11]. Include this in turbo $\mathrm{C}$ then apply the following Transformation algorithm which is a form of computations, can convert the UML Class UML Sequence Diagram.

\section{TRANSFORMATION ALGORITHM 3.1 Process to get Sequence diagram}

As we know that in the UML Class Diagram there are the no of classes, the whole Class Diagram is related to any system, corresponding to every class in the Class Diagram, create an object of that class in the Sequence diagram throughout the whole transformation process [6], [11].

3.1.1 Draw an object

Search for keyword "class" from XML metadata interface file which we have included in turbo C. Using for loop, every time when keyword "class" has been found then create its object in the form of rectangle using $\mathrm{C}$ Graphics function. Code for searching "class" keyword, then placing rectangle as a object in sequence diagram [1], [5].

for $(\mathrm{i}=0 ; \mathrm{i}<=2500 ; \mathrm{i}++)$

\{

$$
\begin{aligned}
& \text { if }\left(\left(\operatorname{ch}[i]=={ }^{\prime} c^{\prime}\right) \& \&(\operatorname{ch}[i+1]==' 1 ') \& \&\left(\operatorname{ch}[i+2]==a^{\prime}\right)\right. \\
& \left.\& \&\left(\operatorname{ch}[i+3]==' s^{\prime}\right) \& \&\left(\operatorname{ch}[i+4]=={ }^{\prime} s^{\prime}\right)\right)
\end{aligned}
$$

\{

rectangle(90,100,170,160);

$$
\mathrm{n}=\mathrm{i} \text {; }
$$

\}

\}

This loop will take the first 2500 characters of the file starting from first character, in loop if condition compare char by char of file with the "class" keyword, by comparing char by char if any where with in the first 2500 characters when found "class" keyword then corresponding automatically create the object of Sequence diagram in the form of rectangle, then again use this loop for finding the next sequence object [4], [5].

3.1.2 Automatic display the name of the class keyword

We know there is name of every class in the Class Diagram, we want the name will be automatically displayed on to the corresponding an object of that class in Sequence diagram [8], [9]. Follow logic as follow

Make use of 2500 characters in for loop starting from first character.

If condition compare char by char from the file and looking for keyword "class",

If the keyword "class" found

Then, we will again search for keyword "name" which is a standard for every UML coding.

If keyword "name" has been found

Then we will start fetching the char from 6th position up to next $14^{\text {th }}$ position i.e. when the name of the class gets fetched.

Repeat these steps for every class name for getting automatically display of name of an object.

\subsubsection{Graphical representation}


The following graphical representation shows graphically that how we have to follow steps to get the automatically fetching of name of the object.

$$
\begin{aligned}
& \operatorname{prn}[\mathrm{j}]=\operatorname{ch}[\mathrm{k}+\mathrm{j}] \\
& \operatorname{cout}<<\operatorname{prn}[\mathrm{j}]
\end{aligned}
$$

$$
\text { \} }
$$

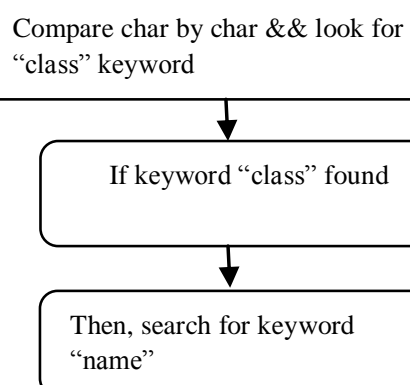

Then, search for keyword "name"

Fig 3: Graphical representation of Transformation Process

\subsubsection{Transformation code}

Following is a code which can be used to get an automatically fetching of the name of an object of the sequence diagram from class diagram

char prn[1000];

for $(\mathrm{i}=0 ; \mathrm{i}<=2500 ; \mathrm{i}++)$

if $\left(\left(\operatorname{ch}[\mathrm{i}]===^{\prime}\right) \& \&\left(\operatorname{ch}[\mathrm{i}+1]==\mathrm{I}^{\prime}\right) \& \&\left(\operatorname{ch}[\mathrm{i}+2]=={ }^{\prime} \mathrm{a}\right) \& \&\right.$ $(\operatorname{ch}[i+3]==' s ') \& \&(\operatorname{ch}[i+4]==' s '))$

\{

for(int $\mathrm{p}=\mathrm{i} ; \mathrm{p}<\mathrm{i}+100 ; \mathrm{p}++)$

\{

if $\left(\left(\operatorname{ch}[\mathrm{p}]==\mathrm{n}^{\prime}\right) \& \&\left(\mathrm{ch}[\mathrm{p}+1]==\mathrm{a}^{\prime}\right) \& \&\right.$ $\left.(\operatorname{ch}[\mathrm{p}+2]==' \mathrm{~m}) \& \&\left(\operatorname{ch}[\mathrm{p}+3]=={ }^{\prime} \mathrm{e}\right)\right)$

\{

int $\mathrm{k}=\mathrm{p}+6$;

for $($ int $j=0 ; j<8 ; j++)$

\{

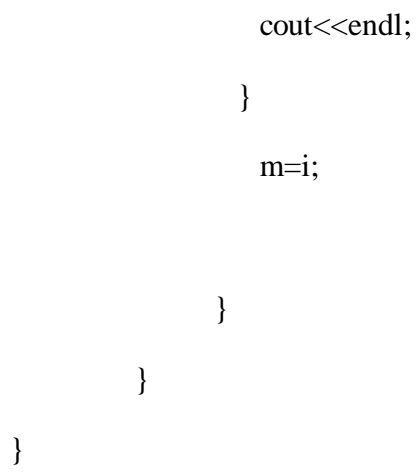

\}

$\mathrm{m}=\mathrm{i}$

\section{FEATURES OF OUR} TRANSFORMATION PROCESS

First, it automatically draws rectangle of an object when a keyword class is found means corresponding to class, there is a rectangle of an object.

Second, it automatically displays a name of an object, when name of a class is found.

\section{COMPARITIVE STUDY OF EXISTING WORK}

There are a many algorithms which can be followed for transforming the UML models to the other form, like the transformation of UML model to Petri Net, research done in [3], [7], In this the UML state chart and UML collaboration, UML activity diagrams are used as an input for the transformation algorithm, which results a Petri Net in the form of Constraints-based Modular Petri Nets, Colored Petri Nets, Extended Colored Petri Nets, and High-Level Timed Petri Nets [3], [5]. But in our research work, we have find out a technique which is used to transform the UML class diagram to UML sequence diagram. This research was not done in previous research [3], [14]

\section{CONCLUSION}

Umbrello software, IBM Rational Software Architect, which can be used to draw any UML model, but these applications are not used to transform the UML class diagram to UML sequence diagram. But in our research work, there is step by step phenomenon for transforming the UML Class Diagram to UML Sequence Diagram. In which there are two main steps Transformation process and Transformation Model. Transformation process used to draw a rectangle for the class object and transformation algorithm displays the automatic name of an object. These features are not available in any software till the date invented. Our approach can be followed to transform the UML class diagram to UML sequence diagram.

\section{FUTURE SCOPE}

This important concept is useful for converting the UML class Diagram to UML sequence diagram conversion. This concept of transformation can be applied to the different models or 
diagrams of the UML, so that there will be automatically transformation takes place from UML class model to its

\section{REFERENCES}

[1] Jeong, Y. J., Cahe, H. S., Chand, C. K. 2011 Semantics based Cohesion and Coupling Metrics for Evaluating Understandability of State Diagram. 35th IEEE Annual Computer Software and Applications Conference.

[2] Mohsin, S., Kaleem, Z., 2010 Program Slicing Based Software Metrics towards code Restructuring. Second international conference on Computer Research and Development, pp. 738-741, 2010

[3] Kerkouche, E., Chaoui, A., Bourennane, B. E., Labbani, O., 2010 "A UML and Colored Petri Nets Integrated Modeling and Analysis Approach using Graph Transformation", Journal of Object Technology Published by ETH Zurich, Chair of Software Engineering, pp25-43.

[4] Haji Ali, N., Shukur, Z. and Idris S. 2007, “Assessment System For UML Class Diagram Using Notations Extraction", IJCSNS International Journal of Computer Science and Network Security, VOL.7No.8, pp181-187.

[5] Kerkouche, E. and Chaoui, A. 2009, "A Formal Framework and a Tool for the Specification and Analysis of G-Nets Models Based on Graph Transformation", International Conference on Distributed Computing and Networking -CDCN'09-, LNCS 5408, pp. 206-211.

[6] Kerkouche, E., Chaoui, A., Bourennane, B. E., and Labbani, O, 2009, "Modeling and verification of Dynamic behaviour in UML models, a graph transformation based approach", proceedings of SEDE, Las Vegas, Nevada, USA, 22-24

[7] Lara, D. J. and Vangheluwe, H. 2002 "AToM3: A Tool for Multi-Formalism Modeling and Meta-Modeling". LNCS 2306, Presented at Fundamental Approaches to Software Engineering - FASE'02, in European Joint Conferences on Theory And Practice of Software ETAPS'02, Grenoble. choice of model or diagram. Our concept is better for conversion from one model to another model.

[8] Lara, D. J. and Vangheluwe, H. 2002, "Computer aided multi-paradigm modeling to process petri-nets and statecharts", International Conference on Graph Transformations (ICGT), Lecture Notes in Computer Science, vol. 2505, pp.239-253.

[9] Lara, D. J. and Vangheluwe, H. (2004), "Meta-Modeling and Graph Grammars for Multi-Paradigm Modeling in AToM3". Software and Systems Modeling, Vol.3, Springer-Verlag, Special Section on Graph Transformations and Visual Modeling Techniques, pp. 194-209.

[10] Murata, T. (1989), "Petri Nets: Properties, Analysis and Applications", Proceedings of the IEEE, Vol. 77, no, pp. 541-580.

[11] Krishnan, Hema, Samuel, Philip.,"Relative Extraction

[12] Methodology for Class Diagram Generation using

[13] Dependency Graph”, ICCCCT, Vol. 10, 1, pp. 815-820,

[14] 2010

[15] Mohsin, Shaikh. Kaleem, Zeeshan,2010, Program Slicing Based Software Metrics towards code Restructuring, Second international conference on Computer Research and Development, pp.738-741.

[16] Blanco, R., Fanjul, J. G. and Tuya, J., 2010, Test case

[17] Generation for transition-pair coverage using Scatter Search. International Journal of Software Engineering and Its Applications Vol. 4, No. 4.

[18] Swain, S. K., Mohapatra, D. P. and Mall, R. 2010. Test

[19] case generation based on state and activity models. Journal of Object Technology, 9(5), pp. 1-27 